\title{
A familial case of alveolar capillary dysplasia with misalignment of the pulmonary veins: the clinicopathological features and unusual glomeruloid endothelial proliferation
}

\author{
Akiko Kitano ${ }^{1}$, Masato Nakaguro², Seiichi Tomotaki ${ }^{3}$, Shintaro Hanaoka³ ${ }^{3}$ Masahiko Kawai ${ }^{3}$, Akiko Saito ${ }^{4}$, \\ Masahiro Hayakawa ${ }^{4}$, Yoshiyuki Takahashi ${ }^{5}$, Hidenori Kawasaki ${ }^{6}$, Takahiro Yamada ${ }^{6}$, Masahiko Ikeda ${ }^{7}$, Tetsuo Onda
} Kazutoshi Cho ${ }^{7}$, Hironori Haga', Atsuko Nakazawa ${ }^{8}$ and Sachiko Minamiguchi ${ }^{1 *}$

\begin{abstract}
Background: Alveolar capillary dysplasia with misalignment of pulmonary veins (ACD/MPV) is a rare disorder of pulmonary vascular abnormality with persistent pulmonary hypertension of the newborn. The symptom usually presents within hours after birth, leading to an early demise. Heterozygous de novo point mutations and genomic deletions of the FOXF1 (forkhead box F1) gene or its upstream enhancer have been identified in most patients with ACD/MPV. Most cases of ACD/MPV are sporadic; however, familial cases are also reported in 10\% of patients.

Case presentation: We herein report a case of familial ACD/MPV that showed unusual glomeruloid proliferation of endothelial cells. In this family, three of the four siblings died within two to 3 days after birth because of persistent pulmonary hypertension and respiratory failure. Only the second child remains alive and healthy. An autopsy was performed for the third and fourth children, resulting in a diagnosis of ACD/MPV based on the characteristic features, including misalignment of smaller pulmonary veins and lymphangiectasis. In both of these children, glomeruloid endothelial proliferation of vessels was noted in the interlobular septa. The vessels were immunohistochemically positive for D2-40, CD31, Factor VIII, and ERG, suggestive of differentiation for both lymphatic and blood vessels.
\end{abstract}

Conclusions: Unusual glomeruloid endothelial proliferation was observed in a familial ACD/MPV case. This histologic feature has not been described previously in ACD/MPV or any other pulmonary disease. Although the histogenesis of this histologic feature is unclear, this finding may suggest that ACD/MPV is a compound vascular and lymphovascular system disorder that exhibits various histologic features.

Keywords: Alveolar capillary dysplasia, Case report, Familial, FOXF1, Glomeruloid endothelial proliferation, Misalignment of pulmonary vein

* Correspondence: minami@kuhp.kyoto-u.ac.jp

'Department of Diagnostic Pathology, Kyoto University Hospital, 54 Kawahara-cho, Sakyo-ku, Kyoto 606-8507, Japan

Full list of author information is available at the end of the article

C The Author(s). 2020 Open Access This article is licensed under a Creative Commons Attribution 4.0 International License, which permits use, sharing, adaptation, distribution and reproduction in any medium or format, as long as you give appropriate credit to the original author(s) and the source, provide a link to the Creative Commons licence, and indicate if changes were made. The images or other third party material in this article are included in the article's Creative Commons licence, unless indicated otherwise in a credit line to the material. If material is not included in the article's Creative Commons licence and your intended use is not permitted by statutory regulation or exceeds the permitted use, you will need to obtain permission directly from the copyright holder. To view a copy of this licence, visit http://creativecommons.org/licenses/by/4.0/ The Creative Commons Public Domain Dedication waiver (http://creativecommons.org/publicdomain/zero/1.0/) applies to the data made available in this article, unless otherwise stated in a credit line to the data. 


\section{Background}

Alveolar capillary dysplasia with misalignment of pulmonary veins $(\mathrm{ACD} / \mathrm{MPV})$ is a rare, fatal developmental disorder of the pulmonary blood vessels. It presents with persistent pulmonary hypertension of the newborn (PPHN) and severe hypoxemia, leading to progressive respiratory failure and an early demise $[1,2]$. PPHN is refractory to all standard medical therapies including extracorporeal membrane oxygenation [3]. The primary characteristic histologic feature of $\mathrm{ACD} / \mathrm{MPV}$ is the anomalous location (misalignment) of smaller pulmonary veins (MPV), which run adjacent to the bronchioles and pulmonary arteries within a common adventitial sheath. In addition, a decreased number of pulmonary capillaries, thickened alveolar septa, medial hypertrophy of small pulmonary arteries, and lymphangiectasis are reported histologic features of $\mathrm{ACD} / \mathrm{MPV}$ in the literature [4-6]. Roughly $50-80 \%$ of patients also show extrapulmonary anomalies in the gastrointestinal, cardiovascular and urogenital systems [1,7]. Genetically, heterozygous de novo point mutations and FOXF1 (forkhead box F1) genomic or its upstream deletion have been reported [8-12]. While many of the reported cases are sporadic, approximately $10 \%$ of $\mathrm{ACD} / \mathrm{MPV}$ cases have a familial association [11-14].

We herein report the clinicopathologic features of a case of familial ACD/MPV exhibiting unusual glomeruloid endothelial proliferation and discuss the significance of these findings.

\section{Case presentation}

The radiographic and macro- and microscopic features of the third and fourth children are shown in Figs. 1, 2 and 3 .

\section{Clinical history}

In this family, three of four siblings born to nonconsanguineous parents died within a few days after birth because of PPHN and respiratory failure. An autopsy was performed for the third and fourth children, and the clinical courses of these cases are described below.

The first child was male and born 5 years prior to the fourth child's birth by Caesarean section due to arrested labor because of cephalopelvic disproportion at 40 weeks' gestation. The mother's age was 30 years old. There were no problems during the pregnancy. The birth weight was $3592 \mathrm{~g}$, and the Apgar scores were 8/9. However, meconium staining of the amniotic fluid was observed at birth, and he was diagnosed with meconium aspiration syndrome. Immediately after birth, he developed hypoxemia, respiratory distress, pneumothorax and severe PPHN. He was treated with mechanical ventilation, supplemental oxygen, chest drainage, pulmonary vasodilators and vasopressor support. However, despite all efforts, he died at $74 \mathrm{~h}$ after birth. An autopsy was not performed for this child.

The second child was male and born 4 years prior to the fourth child's birth by planned Caesarean section at 38 weeks' gestation. The birth weight was $3152 \mathrm{~g}$ and he is alive and healthy without any respiratory problems.

The third child was male and born 2 years prior to the fourth child's birth by planned Caesarean section at 38 weeks' gestation. There were no problems during the pregnancy. The birth weight was $3696 \mathrm{~g}$, and the Apgar scores were 9/10. At about $3 \mathrm{~h}$ after birth, he developed cyanosis and hypoxemia, showing a percutaneous oxygen saturation $\left(\mathrm{SpO}_{2}\right)$ of $60 \%$. His respiratory distress was unresponsive to mechanical ventilation, supplemental oxygen or vasopressor support. He died at $37 \mathrm{~h}$ after birth. An autopsy was performed for this child due to suspicion of congenital interstitial lung diseases.

The fourth child was female and born by planned Caesarean section at 38 weeks' gestation. The mother was 36 years old and had gestational diabetes. The birth weight was $3300 \mathrm{~g}$, and the Apgar scores were 8/8. The infant had postaxial polydactyly on her left foot but no other external malformations. She developed tachypnea at $4 \mathrm{~h}$ after birth, and standard therapies of mechanical ventilation, inhaled nitric oxide and vasopressor support were started. Chest X-ray revealed decreased translucency in the lung field (Fig. 3a). Hypoxemia showed temporary improvement, with the $\mathrm{SpO}_{2}$ increasing to $100 \%$, but she still presented with PPHN. Despite these therapies, she died of respiratory failure at $40 \mathrm{~h}$ after birth, and an autopsy was performed.

Although a familial association was strongly suggested in this case, no similar neonatal deaths had not been observed in other relatives.

\section{Pathologic findings}

An autopsy of the third child was performed $54 \mathrm{~h}$ after his death. The autopsy revealed a male infant measuring $52 \mathrm{~cm}$ in height and weighing $5130 \mathrm{~g}$. No external or internal malformations were detected. Bilateral pleural effusion (100 ml each) and ascites $(350 \mathrm{ml})$ were found, and the left and right lungs weighed $41.6 \mathrm{~g}$ and $49.5 \mathrm{~g}$, respectively. The bilateral lungs were congested and had lost their elasticity (Fig. 1a). Histologically, the diagnosis of $\mathrm{ACD} / \mathrm{MPV}$ was confirmed by the presence of MPV (Fig. 1b). Other characteristic histologic features of $\mathrm{ACD} / \mathrm{MPV}$, such as thickening of alveolar wall (Fig. 1c), hypertrophy of small pulmonary arteries, and lymphangiectasis were also observed. In the interlobular septa, glomeruloid endothelial proliferation was observed in dilated vessels (Figs. 1d-f). The endothelial cells showed no cellular atypia. Although the vessels were more morphologically similar to lymphatic vessels than blood vessels (vessel with thin walls and no or few red blood cells 


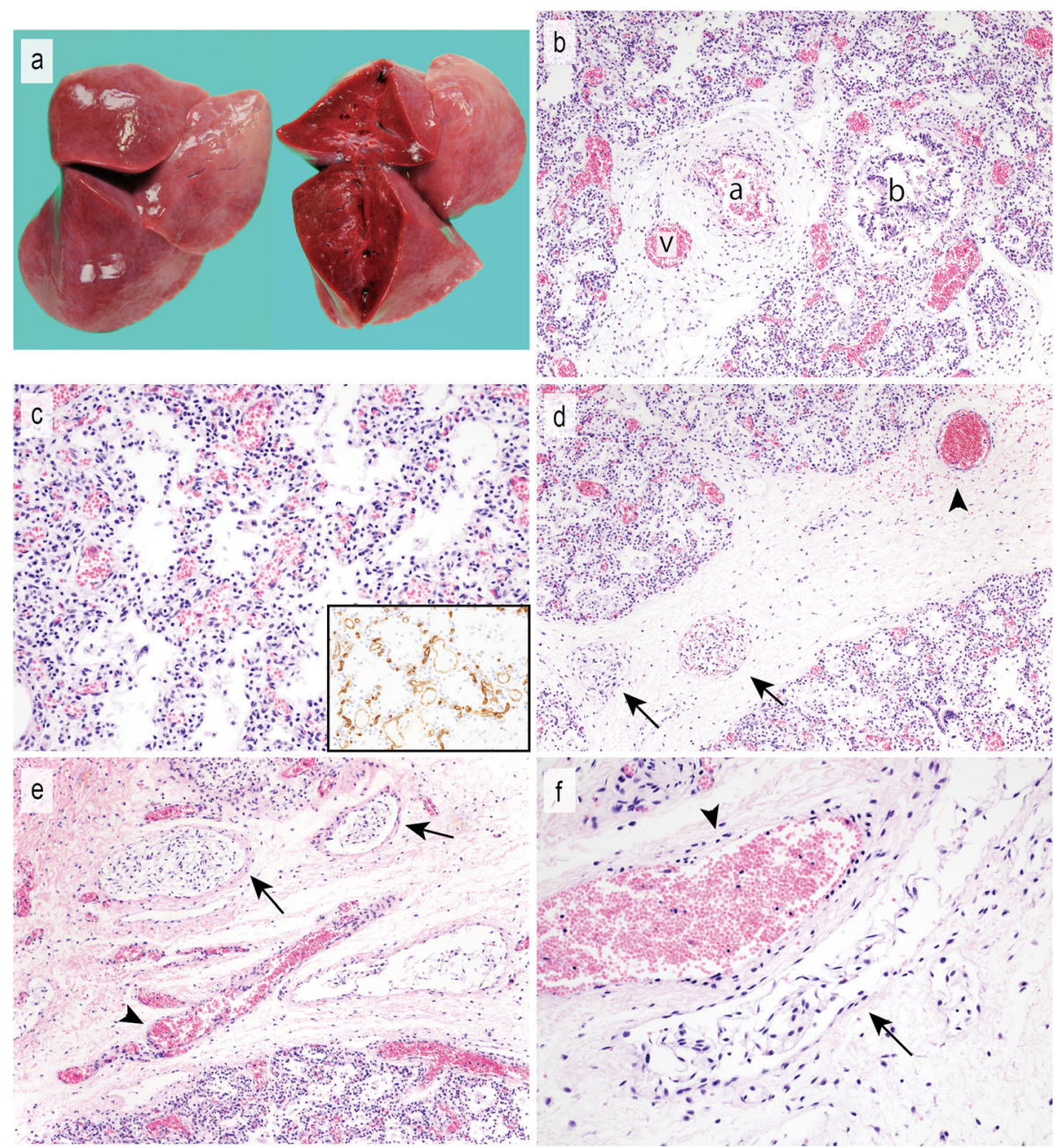

Fig. 1 Histologic and immunohistochemical features of the third child. a A macroscopic image (left) and the cut surface (right) of the right lung before formalin fixation. Congestion and loss of elasticity were noted. $\mathbf{b}$ A misaligned pulmonary vein $(v)$ is adjacent to a pulmonary artery within the same adventitial sheath (a). A bronchiole (b) abuts the vein and artery. c Thickening of the alveolar wall. Immunohistochemical staining for CD34 highlights the dilation of the capillary of the alveolar septa (inset). In this case, a decrease in the number of alveolar capillaries is not remarkable. $\mathbf{d}$-f) Low-power (d, e) and high-power (f) views of the interlobular septum. Vessels with glomeruloid endothelial proliferation (arrow) contrast with blood vessels (arrowhead). The vessels are dilated and contain no or few red blood cells

inside), immunohistochemically, the endothelial cells were positive for D2-40, CD31, Factor VIII, and ERG (Fig. 2). These immunohistochemical results suggested that the vessel had dual differentiation for lymphatic and blood vessels. Blood vessels in the interlobular septa were not remarkable in number, size or structure (Figs. 1d-f). Except for liver congestion, no remarkable findings in other organs were detected.

An autopsy of the fourth child was performed at $82 \mathrm{~h}$ after her death. The autopsy revealed a female infant measuring $47 \mathrm{~cm}$ in height and weighing $3000 \mathrm{~g}$. An external examination showed an extra toe on her left foot. Pleural effusion and ascites were not found, and no internal malformation was identified macroscopically. The left and right lungs weighed $28.0 \mathrm{~g}$ and $33.5 \mathrm{~g}$, respectively, which was within the reference value. In the cut surfaces of the lung, no noticeable findings were observed macroscopically except for small blebs under the pleura of the left upper lung. Histologically, MPV (Figs. 3b, c), a decrease in the number of pulmonary capillaries, thickened alveolar septa (Fig. 3d), lymphangiectasis (Fig. 3e), and medial hypertrophy of small pulmonary arteries were observed in all lobes of the lung. The glomeruloid endothelial proliferation in the interlobular septa that was observed in the third child was present in a limited area (Fig. 3f). Immunohistochemical studies revealed the same results. There were no abnormalities in other organs except for mild liver and splenic congestion. The antibodies used in this study were CD31 (dilution at 1:1200; Dako, Carpinteria, CA), D2-40 (dilution at 1:500; Dako, 


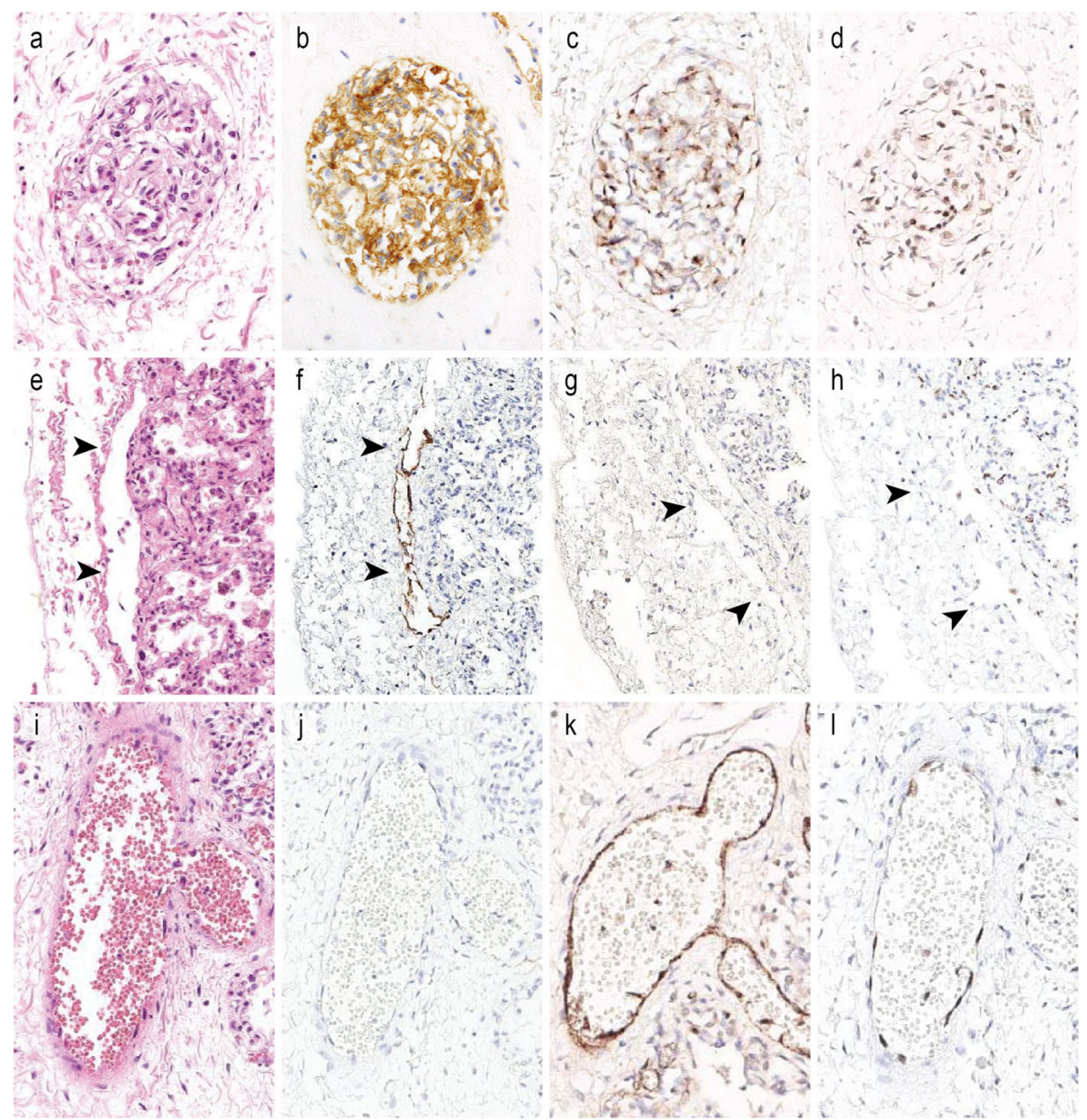

Fig. 2 Immunohistochemical results of glomeruloid endothelial proliferation (a-d), pleural lymphatic vessels (e-h), and blood vessels in interlobular septum (i-I) for D2-40 (b, f, j), Factor VIII (c, g, k), and ERG $(\mathbf{d}, \mathbf{h}, \mathbf{l})$. Glomeruloid endothelial proliferation expressed both lymphatic marker (D2-40) and blood vessel markers (Factor VIII and ERG). (a-d, $\mathbf{i}-\mathbf{l}$ : the third child, $\mathbf{e}-\mathbf{h}$ : another neonatal autopsy case that died from other causes)

Carpinteria, CA), Factor VIII (pre-diluted; Nichirei Biosciences Inc., Tokyo, Japan), and ERG (dilution at 1:100; Abcam, Cambridge, UK).

Tissue samples from the third and fourth children were genetically analyzed. In both cases, a deletion of roughly $450 \mathrm{kbps}$ located about $2840 \mathrm{kbps}$ upstream from exon 1 of the FOXF1 gene was revealed by multiplex ligation-dependent probe amplification (MLPA) assay.

\section{Discussion and conclusions}

$\mathrm{ACD} / \mathrm{MPV}$ is a rare disorder of pulmonary vascular development, and over 200 cases have been reported in the literature [2]. A study reported that the estimated incidence of ACD/MPV was approximately 1/100,000 [2]. However, the true incidence is unclear, as histological examinations by an autopsy or biopsy are necessary in order to confirm the diagnosis. Many patients develop respiratory distress and pulmonary hypertension within the first $24 \mathrm{~h}$ after birth and die within the first month of life. However, there are several case reports of patients with relatively mild ACD/MPV who present with symptoms after $24 \mathrm{~h}$ of life or survive beyond the neonatal period $[1,2,15,16]$. In these milder ACD/MPV patients, the histologic features are reported to be focal or patchy $[15,16]$.

Most ACD/MPV cases are sporadic, but about $10 \%$ involved siblings and a familial association has been suggested [1, 12-14]. In 2009, Stankiewicz et al. suggested an association between ACD/MPV and mutations of the FOX gene cluster on $16 \mathrm{q} 24.1$ [8]. Since then, a number of pathogenic variants containing copy number variations, point mutations and a complex rearrangement have been detected, all involving the FOXF1 gene or its regulatory region [2, 9-12]. While paternal imprinting was suggested in early reports $[8,11]$, variable clinical 

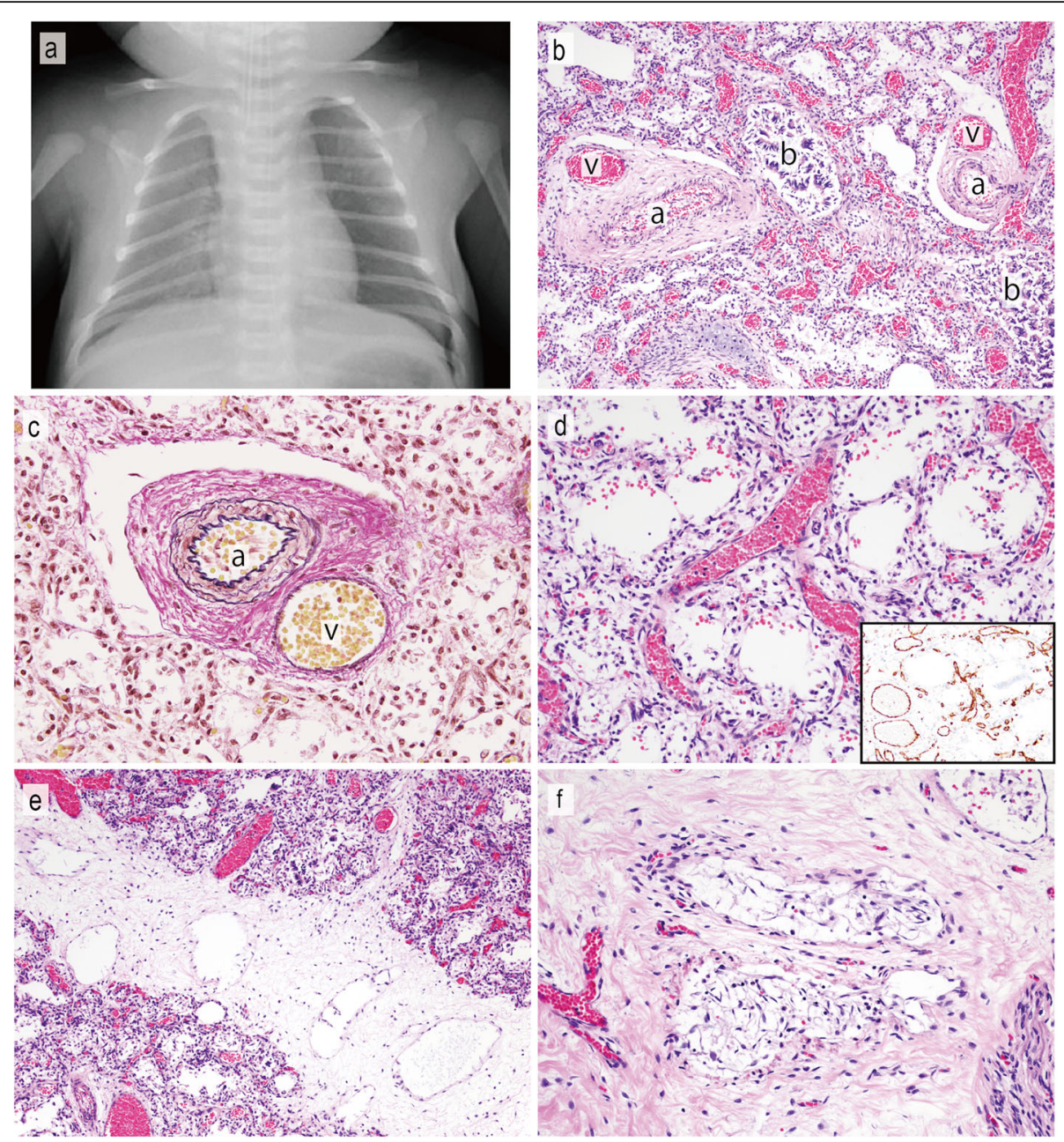

Fig. 3 Radiographic and histopathological findings of the fourth child. a Chest X-ray reveals a diffuse decrease in translucency in the bilateral lung fields. b Misaligned pulmonary veins (v) run alongside the small pulmonary arteries (a) within a common adventitial sheath and a bronchiole (b). c Elastica van Gieson staining highlights the malposition of a pulmonary vein and artery. $\mathbf{d}$ Thickening of the alveolar wall and dilation of vessels. Immunohistochemical staining for CD31 shows a decreased number of pulmonary capillaries located away from the alveolar epithelium (inset). e Most of the lymphatic vessels in the interlobular septa are dilated without endothelial proliferation, and glomeruloid endothelial proliferation was limited to a small area (f)

presentations in a single family with the same FOXF1 mutation have suggested somatic mosaicism and complex gene regulation, including unorthodox imprinting of the gene locus [12]. In the present case, we performed genetic testing for the third and fourth children and found the identical deletion of the upstream enhancer region of the FOXF1 gene. This deletion was previously reported in ACD/MPV patients and included the fetal lung-expressed long noncoding RNA gene LINC01081, which positively regulates the FOXF1 expression [17].

$\mathrm{ACD} / \mathrm{MPV}$ is histopathologically characterized and defined by the presence of MPV [2]. Other histologic features include a decrease in the number of pulmonary capillaries, thickening of alveolar septa, lymphangiectasis, and medial hypertrophy of small pulmonary arteries and muscularization. In the present case, we found these typical histologic features in both children. In addition, glomeruloid endothelial proliferation was observed in the vessels in the interlobular septa (Figs. 1d-f, Fig. 3f). The immunohistochemical results were difficult to interpret. While the pleural lymphatic vessels in the present cases and two other neonatal autopsy cases that died of other causes were immunoreactive for D2-40 and CD31 and negative for Factor VIII and ERG (Figs. 2e-h), the proliferated endothelium was positive for all of these markers (Figs. 2a-d). These results suggested that the vessels could not be simply classified as lymphatic or blood vessels, instead showing differentiation for both lymphatic and blood vessels. These double (lymphatic and blood vessel markers)-positive vessels were reported in a previous study of gastric adenocarcinoma [18]. Although the possibility of immature vasculature was 
suggested, the origin and significance has not still been elucidated [18]. The vessels of the present cases had thinner walls and no or fewer red blood cells inside than typical blood vessels (Figs. 1d-f, Fig. 3f). The morphology thus appears to be more similar to lymphatic vessels than blood vessels. The previous report of doublepositive vessels also found them to be morphologically similar to lymphatic vessels [18]. This unusual glomeruloid endothelial proliferation has not been reported previously in $\mathrm{ACD}$ or, to our knowledge, any other pulmonary disorders or neonatal lung.

We compared the distribution and number of lymphatic vessels with the aforementioned two neonate autopsy cases that died from other causes. The number and morphology of pleural lymphatic vessel were not markedly different between the present cases and the non-ADC/ MPV autopsy cases. However, in the present cases, the interlobular and intralobular lymphatic vessels, particularly the bronchovascular lymphatic vessels, were dilated and more prominent on D2-40 immunohistochemical staining than non-ACD/MPV cases. This was, although not quantified, caused by the dilation and increase in tortuosity of the lymphatic vessels.

As the histogenesis of the glomeruloid endothelial proliferation, both the possibility of reactive endothelial hyperplasia and primary change caused by ACD/MPV were considered. Given the identical genetic deletion in both children, it was suggested that the lesion was primarily caused by FOXF1 alteration and that ACD/MPV is a compound vascular and lymphovascular system disorder exhibiting divergent histologic features.

Extrapulmonary congenital anomalies, including cardiovascular, gastrointestinal, and genitourinary systems, have been reported in both sporadic and familial ACD/ MPV cases [11]; however, in the present cases, no symptomatic congenital anomalies were detected except for an extra toe in the fourth child.

At present, the definitive diagnosis of $\mathrm{ACD} / \mathrm{MPV}$ is made by a histologic examination of the lungs, typically at a postmortem autopsy [5]. However, the diagnosis based on a lung biopsy has also been reported recently $[16,19,20]$. In order to confirm the presence of MPV, it is necessary to collect specimens, including blood vessels, by an open lung biopsy. In our case, MPV were distributed diffusely in all lobes, even in the peripheral area ( $3 \mathrm{~mm}$ from the pleura) and an open biopsy could have made a definitive diagnosis if the specimen had been larger than $3 \mathrm{~mm}$ in size. However, the clinical course was urgent and a biopsy could not be performed.

In conclusion, we reported a familial case of $\mathrm{ACD} /$ MPV with unusual glomeruloid endothelial proliferation. Careful histologic evaluation on lymphatic vessels in future cases and further basic studies are warranted to understand the pathogenesis of ACD/MPD.

\section{Abbreviations}

ACD: Alveolar capillary dysplasia; FOXF1: Forkhead box F1;

MPV: Misalignment of pulmonary veins; PPHN: Persistent pulmonary hypertension of the newborn; $\mathrm{SpO}_{2}$ : Percutaneous oxygen saturation

\section{Acknowledgements}

Not applicable.

\section{Authors' contributions}

All authors contributed to the scientific discussion and final approval of the manuscript. AK, MN, AN and SM contributed to conception and pathological evaluation. ST, SH, MK, AS, MH, YT, HK, and TY contributed to clinical evaluation. MI, TO, and KC performed genetic analysis. AK, MN, and SM wrote the draft of the manuscript.

\section{Funding}

Not applicable.

\section{Availability of data and materials}

All data generated or analyzed during this study are included in this published article.

\section{Ethics approval and consent to participate}

Not applicable.

\section{Consent for publication}

Written informed consent was obtained from the patients' parents or representative person for publication of this case report and any accompanying images. A copy of the written consent is available for review by the Editor of this journal.

\section{Competing interests}

The authors declare that they have no competing interests.

\section{Author details}

${ }^{1}$ Department of Diagnostic Pathology, Kyoto University Hospital, 54 Kawahara-cho, Sakyo-ku, Kyoto 606-8507, Japan. 'Department of Pathology and Laboratory Medicine, Nagoya University Hospital, Nagoya, Japan. ${ }^{3}$ Department of Pediatrics, Graduate School of Medicine, Kyoto University, Kyoto, Japan. ${ }^{4}$ Division of Neonatology, Center for Maternal-Neonatal Care, Nagoya University Hospital, Nagoya, Japan. ${ }^{5}$ Department of Pediatrics, Nagoya University Graduate School of Medicine, Nagoya, Japan. ${ }^{6}$ Clinical Genetics Unit, Kyoto University Hospital, Kyoto, Japan. ${ }^{7}$ Maternity and Perinatal Care Center, Hokkaido University Hospital, Sapporo, Japan.

${ }^{8}$ Department of Clinical Research, Saitama Children's Medical Center, Saitama, Japan.

Received: 7 November 2019 Accepted: 5 May 2020

Published online: 09 May 2020

\section{References}

1. Bishop NB, Stankiewicz P, Steinhorn RH. Alveolar capillary dysplasia. Am J Respir Crit Care Med. 2011;184(2):172-9.

2. Slot E, Edel G, Cutz E, van Heijst A, Post M, Schnater M, et al. Alveolar capillary dysplasia with misalignment of the pulmonary veins: clinical, histological, and genetic aspects. Pulm Circ. 2018;8(3):2045894018795143.

3. Michalsky MP, Arca MJ, Groenman F, Hammond S, Tibboel D, Caniano DA Alveolar capillary dysplasia: a logical approach to a fatal disease. J Pediatr Surg. 2005:40(7):1100-5.

4. Janney CG, Askin FB, Kuhn C 3rd. Congenital alveolar capillary dysplasia--an unusual cause of respiratory distress in the newborn. Am J Clin Pathol. 1981; 76(5):722-7.

5. Sen P, Thakur N, Stockton DW, Langston C, Bejjani BA. Expanding the phenotype of alveolar capillary dysplasia (ACD). J Pediatr. 2004;145(5):64651.

6. Singh SA, Ibrahim T, Clark DJ, Taylor RS, George DH. Persistent pulmonary hypertension of newborn due to congenital capillary alveolar dysplasia. Pediatr Pulmonol. 2005:40(4):349-53.

7. Nguyen L, Riley MM, Sen P, Galambos C. Alveolar capillary dysplasia with misalignment of pulmonary veins with a wide spectrum of extrapulmonary manifestations. Pathol Int. 2013;63(10):519-21. 
8. Stankiewicz P, Sen P, Bhatt SS, Storer M, Xia Z, Bejjani BA, et al. Genomic and genic deletions of the FOX gene cluster on 16q24.1 and inactivating mutations of FOXF1 cause alveolar capillary dysplasia and other malformations. Am J Hum Genet. 2009:84(6):780-91.

9. Sen P, Yang Y, Navarro C, Silva I, Szafranski P, Kolodziejska KE, et al. Novel FOXF1 mutations in sporadic and familial cases of alveolar capillary dysplasia with misaligned pulmonary veins imply a role for its DNA binding domain. Hum Mutat. 2013;34(6):801-11.

10. Szafranski P, Yang Y, Nelson MU, Bizzarro MJ, Morotti RA, Langston C, et al. Novel FOXF1 deep intronic deletion causes lethal lung developmental disorder, alveolar capillary dysplasia with misalignment of pulmonary veins. Hum Mutat. 2013;34(11):1467-71.

11. Sen P, Gerychova R, Janku P, Jezova M, Valaskova I, Navarro C, et al. A familial case of alveolar capillary dysplasia with misalignment of pulmonary veins supports paternal imprinting of FOXF1 in human. Eur J Hum Genet. 2013;21(4):474-7.

12. Reiter J, Szafranski P, Breuer O, Perles Z, Dagan T, Stankiewicz P, et al. Variable phenotypic presentation of a novel FOXF1 missense mutation in a single family. Pediatr Pulmonol. 2016;51(9):921-7.

13. Boggs S, Harris MC, Hoffman DJ, Goel R, McDonald-McGinn D, Langston C, et al. Misalignment of pulmonary veins with alveolar capillary dysplasia: affected siblings and variable phenotypic expression. J Pediatr. 1994;124(1): $125-8$.

14. Gutierrez C, Rodriguez A, Palenzuela S, Forteza C, Rossello JL. Congenital misalignment of pulmonary veins with alveolar capillary dysplasia causing persistent neonatal pulmonary hypertension: report of two affected siblings. Pediatr Dev Pathol. 2000;3(3):271-6.

15. Towe CT, White FV, Grady RM, Sweet SC, Eghtesady P, Wegner DJ, et al. Infants with atypical presentations of alveolar capillary dysplasia with misalignment of the pulmonary veins who underwent bilateral lung transplantation. J Pediatr. 2018;194:158-64.e1.

16. Edwards JJ, Murali C, Pogoriler J, Frank DB, Handler SS, Deardorff MA, et al. Histopathologic and genetic features of alveolar capillary dysplasia with atypical late presentation and prolonged survival. J Pediatr. 2019;210:214.

17. Szafranski P, Gambin T, Dharmadhikari AV, Akdemir KC, Jhangiani SN, Schuette J, et al. Pathogenetics of alveolar capillary dysplasia with misalignment of pulmonary veins. Hum Genet. 2016;135(5):569-86.

18. Sasaki H, Morohashi S, Toba T, Seino H, Yoshizawa T, Hirai H, et al. Neoangiogenesis of gastric submucosa-invasive adenocarcinoma. Oncol Lett. 2018;16(3):3895-900

19. Castilla-Fernandez Y, Copons-Fernandez C, Jordan-Lucas R, Linde-Sillo A, Valenzuela-Palafoll I, Ferreres Pinas JC, et al. Alveolar capillary dysplasia with misalignment of pulmonary veins: concordance between pathological and molecular diagnosis. J Perinatol. 2013;33(5):401-3.

20. Eulmesekian P, Cutz E, Parvez B, Bohn D, Adatia I. Alveolar capillary dysplasia: a six-year single center experience. J Perinat Med. 2005;33(4):34752

\section{Publisher's Note}

Springer Nature remains neutral with regard to jurisdictional claims in published maps and institutional affiliations.

Ready to submit your research? Choose BMC and benefit from:

- fast, convenient online submission

- thorough peer review by experienced researchers in your field

- rapid publication on acceptance

- support for research data, including large and complex data types

- gold Open Access which fosters wider collaboration and increased citations

- maximum visibility for your research: over $100 \mathrm{M}$ website views per year

At $\mathrm{BMC}$, research is always in progress.

Learn more biomedcentral.com/submissions 